\title{
Influence of Electrolyte Type, pH, Temperature and Aging on the Viscosity Property of Okra Gum as a Suspending Agent in Paracetamol Suspension
}

\author{
Vincent Obaga Nyandoro*, Joshua Ikoni Ogaji, Jennifer Drambi Audu-Peter
}

Department of Pharmaceutical Technology and Industrial Pharmacy, Faculty of Pharmaceutical Sciences, University of Jos, PMB 2084 Jos 93003, Plateau State, Nigeria

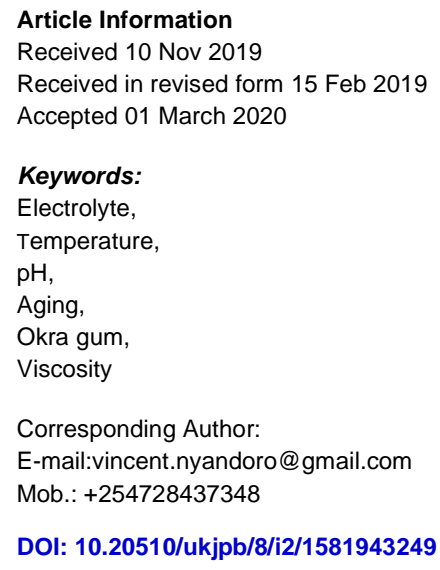

\begin{abstract}
The purpose of this study was to investigate the influence of electrolyte type, $\mathrm{pH}$, temperature and aging on the viscosity property of okra gum using paracetamol as model drug. Paracetamol (125 mg/ $5 \mathrm{~mL}$ ) suspension containing okra gum particles of undersize $180 \mu \mathrm{m}$ as suspending agent was formulated. Similar suspension of paracetamol was formulated using tragacanth gum as a suspending agent for comparison. Effect of electrolyte type, $\mathrm{pH}$, temperature and aging on the viscosity property of okra gum in paracetamol suspension was evaluated using standard methods. Addition of electrolytes, changes in $\mathrm{pH}$, increase in temperature and increase in storage time were shown to decrease the viscosity of the paracetamol suspension. The effect of temperature and aging on the viscosity of the suspension formulation containing okra gum was more pronounced than on the formulation containing tragacanth gum. Conversely, the effect of alkaline $\mathrm{pH}$ and type of electrolyte on the viscosity of the suspension formulation containing tragacanth gum was more pronounced than on the formulation containing okra gum. On the basis of these, formulators and caregivers may consider these factors when designing or using pharmaceutical suspensions containing natural gums such as okra and tragacanth as viscosity enhancing agents.
\end{abstract}

\section{Introduction}

Viscosity is termed as resistance to flow ${ }^{1}$. Viscosity of a pharmaceutical suspension is of great importance because it affects stability, redispersibility, drug release and pourability of a suspension ${ }^{2}$. A good suspension should have adequate viscosity to ensure that the dispersed drug particles remain suspended long enough to maintain stability ${ }^{3}$.

A good suspension should also have reasonable viscosity to ensure that drug release process is not impeded. A study by Venkateswarlu, Chnadrasekhar and Ramachandra ${ }^{4}$ found out that drug release from flucloxacillin suspension decreased as viscosity increased due to formation of high viscosity regions on the layer surrounding drug particles and in the bulk medium due to reduced diffusion process caused by hydrated polymer chains. The ease with which a suspension pours during withdrawal depends on the viscosity of the suspension; with less viscous pouring more easily than more viscous suspensions ${ }^{5}$. Adequate viscosity ensures the caregiver is able to pour the suspension from the packaging container without difficult during administration.

The viscosity of a medium is not constant and factors such as concentration of suspending agent, $\mathrm{pH}$ changes, electrolytes (ionic strength), sugars, ageing, temperature and type of agitation have been reported to affect viscosity of a suspension ${ }^{6}$. It is important to know how these factors affect viscosity since alteration of viscosity influences other physicochemical properties such as stability, redispersibility, drug release and pourability of a suspension thus affecting performance of the suspension.

Paracetamol is sparingly soluble $(14 \mathrm{mg} / \mathrm{mL}$ ) in aqueous medium. This means that only $70 \mathrm{mg}$ of the paracetamol powder will dissolve in $5 \mathrm{ml}$ of water. The pediatric paracetamol dose of 
$125 \mathrm{mg} / 5 \mathrm{~mL}$ with require that some of the paracetamol powder is suspended in water thus paracetamol is a candidate for formulation as a suspension. It is an indiffusible solid ${ }^{7}$ that does not remain suspended for enough time to allow withdrawal of uniform doses. Therefore, to formulate paracetamol suspension, a suspending agent such as okra gum is required to increase the viscosity of the disperse system, reduce settling of the suspended drug particles, maintain uniform dispersion and prevent cake formation ${ }^{3}$.

Okra gum has been used as suspending agent in pharmaceutical suspension formulations ${ }^{7,8}$. However, it is not known how electrolytes, temperature, $\mathrm{pH}$ and aging can affect the viscosity property of okra gum when used in these formulations. Therefore, the study sought to provide knowledge on the influence of these properties on the viscosity property of okra gum when used in a formulation, an important aspect in the development and use of pharmaceutical suspensions.

\section{Materials and methods}

\subsection{Materials}

Okra gum (extracted), Tragacanth gum (Lab Tech Chemicals), Paracetamol powder (A.H.A International Co. Ltd, Batch No. $Y$ 142870). All chemical and reagents used were of analytical grade.

\subsection{Methods}

\subsubsection{Collection and Identification of Okra Plant Pods}

Okra pods were obtained from Faringada market at Jos, Plateau State in September, 2018. Whole okra plant with pods was taken to a botanist at the College of Forestry, Jos for identification and the herbarium voucher number was FHJ 241.

\subsubsection{Extraction and Purification of Okra Gum}

The method of Farooq, Malviya and Sharma ${ }^{9}$ was adopted. Fresh Okra pods were washed with purified water, sliced, air-dried in the laboratory and weighed. The dried sliced okra was macerated in cold water for 24 hours to extract the mucilage and the dispersion was separated from the chaff using a muslin cloth. The mucilage was centrifuged (Centrifuge, Mistral 1000, UK) at 4500 rpm for ten minutes and then treated severally with $96 \%$ ethanol to precipitate the gum. The gum was air dried, pulverized, weighed and packaged in a well-closed container for subsequent use. The percentage yield of the gum from dried okra was calculated using equation;

Percentage yield $=\frac{\text { Mass of dry okra gum x } 100 \%}{\text { Mass of dry sliced okra pods }}$

(Equation 1)

The powdered okra gum was passed through sieve undersize $180 \mu \mathrm{m}$ to obtain undersize $180 \mu \mathrm{m}$ particle size distribution.
Tragacanth gum was treated in similar way and used for comparison.

\subsubsection{Preparation of Oral Paracetamol Suspension}

The method of Venkateswarlu, Chnadrasekhar and Ramachandra ${ }^{4}$ was adopted. A paracetamol suspension to deliver a dose of $125 \mathrm{mg} / 5 \mathrm{~mL}$ was formulated using $0.6 \% \mathrm{w} / \mathrm{v}$ of okra gum and tragacanth gum as suspending agents. Desired quantities of paracetamol powder and excipient powders (Table 1) were finely ground, screened through a sieve of undersize 180 $\mu \mathrm{m}$ and weighed.

Sodium lauryl sulphate was mixed with sodium citrate in a mortar using a pestle for two minutes to form a mix. To the mix, sodium benzoate was added and mixed for two minutes. Gum powder was added to mix and mixed for three minutes. Paracetamol powder was added to the mix and mixed for three minutes to form a blend of dry powder suspension. A $100 \mathrm{~mL}$ of water was added in small quantities while mixing until a suspension was formed. The components of the oral reconstitutable suspensions are shown in table 1.

Table 1: Formulation Components of Paracetamol Suspension

\begin{tabular}{lcc}
\hline Ingredient $(\% \mathbf{w} / \mathbf{v})$ & \multicolumn{2}{c}{ Batch } \\
\cline { 2 - 3 } & S1 & S2 \\
\hline Paracetamol & 2.5 & 2.5 \\
Okra gum powder & 0.6 & - \\
Tragacanth gum & - & 0.6 \\
Sodium benzoate & 0.5 & 0.5 \\
Sodium citrate & 0.5 & 0.5 \\
Sodium lauryl sulphate & 0.02 & 0.02 \\
\hline
\end{tabular}

\subsubsection{Evaluation of Oral Paracetamol Suspension}

\subsubsection{Viscosity determination}

The method of Ogaji ${ }^{7}$ was adopted. Viscosity of the suspension was determined after reconstitution using a digital viscometer, (model NDJ-1S, Shandong, China) at $30 \mathrm{rpm}$. A $50 \mathrm{~mL}$ of the suspension was transferred into the viscometer cup and after one min, viscosity was determined using rotor 2 spindle. The result was in triplicate and expressed as mean values

\subsubsection{2 $\mathrm{pH}$ determination}

The method of Emeje et $\mathrm{al}^{10}$ was adopted. The $\mathrm{pH}$ of the reconstituted paracetamol suspensions at room temperature was determined as previously described using a digital $\mathrm{pH}$ meter (Setra, PHS-25, China). 


\subsubsection{Effect of $\mathrm{pH}$ on viscosity of the paracetamol suspension}

The method of Kadiri and Okafor ${ }^{11}$ was adopted. A $1 \mathrm{~mL}$ of $0.1 \mathrm{~N}$ hydrochloric acid $(\mathrm{HCl})$ was added to the formulated paracetamol suspension and the suspension was made into $50 \mathrm{~mL}$. The viscosity of the suspension was determined using a digital rotational viscometer. The mean of the three determinations was recorded. The same process was repeated using $1 \mathrm{~mL}$ of $0.1 \mathrm{~N}$ sodium hydroxide $(\mathrm{NaOH})$.

2.2.4.4 Effect of temperature on viscosity of the paracetamol suspension

The method of Kadiri and Okafor ${ }^{11}$ was adopted. A $50 \mathrm{~mL}$ of the suspension was transferred into the viscometer cup and after one minute, viscosity was determined using rotor 2 spindle. The suspensions were then stored in a refrigerator (HR 170T) at $4{ }^{\circ} \mathrm{C}$ and in a dryer (Gallenkamp drying cabinet, Germany) at $40^{\circ} \mathrm{C}$ for twenty-four hours and the viscosity of the suspensions were measured using a digital rotational viscometer. The mean of the three determinations was recorded.

2.2.4.5 Effect of electrolyte type on viscosity of the paracetamol suspension

The method of Kadiri and Okafor ${ }^{11}$ was adopted. A $1 \mathrm{~mL}$ of $0.5 \%$ $\mathrm{w} / \mathrm{v}$ sodium chloride, calcium chloride or aluminium chloride was added to $50 \mathrm{~mL}$ of the formulated paracetamol suspensions and allowed to stand for twenty-four (24) hours. The viscosity of the suspensions was measured using a digital rotational viscometer. The mean of the three determinations was recorded.

\subsubsection{Effect of ageing on viscosity of the paracetamol} suspension

The method of Kadiri and Okafor ${ }^{11}$ was adopted. A $50 \mathrm{~mL}$ of the paracetamol suspension was transferred into the viscometer cup and viscosity was determined using Rotor 2 spindle. The suspension was then stored at room temperature for two months and the viscosity of the suspension was measured using a digital rotational viscometer. The mean of the three determinations was recorded.

\section{Results and discussion}

\subsection{Effect of $\mathrm{pH}$ change on viscosity of paracetamol suspension}

Table 2 shows the effect of $\mathrm{pH}$ on viscosity of paracetamol suspensions formulated using okra gum and tragacanth gum particles of undersize $180 \mu \mathrm{m}$. Both alkaline $(0.1 \mathrm{~N} \mathrm{NaOH})$ and acidic $\mathrm{pH}(0.1 \mathrm{~N} \mathrm{HCl})$ caused a drop in viscosity of the suspensions. The viscosity of the suspension containing okra gum was more affected by acidic $\mathrm{pH}$ than alkaline $\mathrm{pH}$. The viscosity dropped by $5.24 \%$ and $3.36 \%$ in acidic $\mathrm{pH}$ and alkaline $\mathrm{pH}$ respectively. On the other hand, the viscosity of the suspension containing tragacanth gum was more affected by alkaline $\mathrm{pH}$ than acidic $\mathrm{pH}$; dropping by $4.90 \%$ and $3.24 \%$ respectively.
The viscosity of the paracetamol suspension containing okra gum, a weak acid, dropped by $5.24 \%$ compared to the viscosity of the paracetamol suspension containing tragacanth gum, a weak base which dropped by $3.24 \%$. On the other, suspensions containing weakly basic gums are more affected by strong bases such as sodium hydroxide. The viscosity of the paracetamol suspension containing tragacanth gum, a weak base, dropped by $4.90 \%$ compared to the viscosity of the paracetamol suspension containing okra gum, a weak acid which dropped by $3.36 \%$. These findings augment the findings by Kadiri and Okafor ${ }^{11}$ which found out that the impact of changes in $\mathrm{pH}$ on the viscosity of the medium depends on the initial $\mathrm{pH}$ and suspensions containing weakly acidic gums are greatly affected by strong acids such as hydrochloric acid.

Table 2: Effect of pH change on Viscosity of Paracetamol Suspension

\begin{tabular}{lcc}
\hline Parameter & S1 & S2 \\
\hline pH & & \\
Initial & $6.68 \pm 0.02$ & $7.40 \pm 0.01$ \\
After addition of $0.1 \mathrm{~N}$ & $7.08 \pm 0.01$ & $7.70 \pm 0.01$ \\
$\mathrm{NaOH}$ & &
\end{tabular}

Viscosity in mpa.s

Initial

$218.00 \pm 3.46$

$674.67 \pm 8.37$

After addition of $0.1 \mathrm{~N}$

$210.67 \pm 0.58$

$641.67 \pm 6.81$

$\mathrm{NaOH}$

$\%$ change

$\downarrow 3.36$

$\downarrow 4.90$

pH

$\begin{array}{lll}\text { Initial } & 6.68 \pm 0.01 & 7.41 \pm 0.02 \\ \text { After addition of } 0.1 \mathrm{~N} & 6.25 \pm 0.01 & 6.89 \pm 0.02 \\ \mathrm{HCl} & & \end{array}$

Viscosity in mpa.s

Initial

$222.67 \pm 0.58 \quad 678.33 \pm 6.03$

After addition of $0.1 \mathrm{~N} \quad 211.00 \pm 2.65 \quad 656.33 \pm 8.96$

$\mathrm{HCl}$

$\%$ change

$\downarrow 5.24$

$\downarrow 3.24$

Mean $\pm S D(n=3$ determinations $)$

3.2 Effect of temperature on viscosity of paracetamol suspension

Table 3 shows the effect of temperature on viscosity of paracetamol suspensions formulated using okra gum and tragacanth gum particles of undersize $180 \mu \mathrm{m}$. Viscosity of the both suspensions decreased with increase in temperature and vice versa. At $4{ }^{\circ} \mathrm{C}$, both suspensions solidified. At $40{ }^{\circ} \mathrm{C}$, the viscosity of the suspensions dropped by $12.96 \%$ and $9.42 \%$ for suspensions containing okra gum and tragacanth gum 
respectively. The drop in viscosity was more in the suspension containing okra gum than in the suspension containing tragacanth gum.

Table 3: Effect of Temperature on Viscosity of Paracetamol Suspension

\begin{tabular}{lccc}
\hline \multirow{2}{*}{$\begin{array}{l}\text { Formula } \\
\text { tion }\end{array}$} & \multicolumn{3}{c}{ Viscosity in mpa.s } \\
\cline { 2 - 4 } & $\mathbf{2 7}{ }^{\circ} \mathbf{C}$ (Room temp) & $\mathbf{4}^{\circ} \mathbf{C}$ & $\%$ change \\
\hline S1 & $235.00 \pm 3.464$ & Solidified & NA \\
S2 & $676.00 \pm 3.61$ & Solidified & NA \\
& $\mathbf{2 7}{ }^{\circ} \mathbf{C}$ (Room temp) & $\mathbf{4 0}{ }^{\circ} \mathbf{C}$ & $\%$ change \\
\cline { 2 - 4 } S1 & $218.00 \pm 3.00$ & $190.33 \pm 0.58$ & $\downarrow 12.69$ \\
S2 & $654.67 \pm 3.79$ & $593.00 \pm 7.00$ & $\downarrow 9.42$ \\
\hline
\end{tabular}

Mean \pm SD ( $\mathrm{n}=3$ determinations $)$

Viscosity of the paracetamol suspensions decreased with increase in temperature and increased with decrease in temperature augmenting the previous finding by Mudgil et $\mathrm{al}^{6}$. Decrease in viscosity with temperature indicated that the heat exerted a viscosity thinning effect on the paracetamol suspensions. This was possibly due to loss of water molecules around the suspending agent particles when the temperature increased leading to decreased viscosity of the suspensions. Increase in temperature also reduces frictional force between molecules resulting in decrease in viscosity. Another possible explanation could be that the heat increased the energy and movement of water molecules thereby decreasing intermolecular interactions making the water molecules to lose their ordering around the suspending agent particles and thus affected their conformation consequently resulting in reduced viscosity Venkateswarlu, Chnadrasekhar and Ramachandra ${ }^{4}$.

3.3 Effect of type of electrolyte on viscosity of paracetamol suspension

Table 4 shows the effect of type of electrolyte on viscosity of paracetamol suspensions formulated using okra gum and tragacanth gum particles of undersize $180 \mu \mathrm{m}$. Addition of sodium chloride $(0.5 \% \mathrm{NaCl})$, calcium chloride $\left(0.5 \% \mathrm{CaCl}_{2}\right)$ and aluminium chloride $\left(0.5 \% \mathrm{AlCl}_{3}\right)$ electrolytes led to decrease in viscosity of the suspensions. There was an inverse relationship between viscosity and the valence of the electrolytes as exhibited by more decrease in viscosity as the valence of the electrolyte increased. $\mathrm{AlCl}_{3}$ was more effective in decreasing viscosity (decreased viscosity by $12.93 \%$ ) than $\mathrm{CaCl}_{2}$ (decreased viscosity by $8.02 \%$ ) which in turn was more effective than $\mathrm{NaCl}$ (decreased viscosity by $6.38 \%$ ).

Addition of electrolytes to the paracetamol suspensions decreased their viscosity and the impact increased as the charge on the ion increased from monovalent to trivalent. This is possibly due to electrolytes changing the charge density thus affecting the intermolecular interactions. These findings agrees to early findings by Kadiri and Okafor ${ }^{11}$ which reported that addition of electrolytes to metronidazole suspension decreased the viscosity.

Table 4: Effect of Electrolyte Type on Viscosity of Paracetamol Suspension

\begin{tabular}{|c|c|c|}
\hline Parameter & S1 & S2 \\
\hline \multicolumn{3}{|l|}{ pH } \\
\hline Initial & $6.64 \pm 0.01$ & $7.32 \pm 0.02$ \\
\hline After addition of $0.5 \% \mathrm{NaCl}$ & $6.58 \pm 0.02$ & $7.33 \pm 0.01$ \\
\hline \multicolumn{3}{|l|}{ Viscosity in mpa.s } \\
\hline Initial & $235.00 \pm 1.00$ & $716.00 \pm 436$ \\
\hline After addition of $0.5 \% \mathrm{NaCl}$ & $220.00 \pm 0.00$ & $652.00 \pm 3.61$ \\
\hline$\%$ change & $\downarrow 6.38$ & $\downarrow 8.94$ \\
\hline \multicolumn{3}{|l|}{ pH } \\
\hline Initial & $6.64 \pm 0.01$ & $7.31 \pm 0.01$ \\
\hline After addition of $0.5 \%$ & $6.62 \pm 0.01$ & $7.30 \pm 0.02$ \\
\hline \multicolumn{3}{|l|}{$\mathrm{CaCl}_{2}$} \\
\hline \multicolumn{3}{|l|}{ Viscosity in mpa.s } \\
\hline Initial & $195.33 \pm 1.53$ & $711.67 \pm 13.61$ \\
\hline After addition of $0.5 \%$ & $179.67 \pm 1.15$ & $624.33 \pm 1.53$ \\
\hline \multicolumn{3}{|l|}{$\mathrm{CaCl}_{2}$} \\
\hline$\%$ change & $\downarrow 8.02$ & $\downarrow 12.27$ \\
\hline \multicolumn{3}{|l|}{ pH } \\
\hline Initial & $6.64 \pm 0.01$ & $7.36 \pm 0.02$ \\
\hline After addition of $0.5 \% \mathrm{AlCl}_{3}$ & $6.61 \pm 0.01$ & $7.36 \pm 0.01$ \\
\hline \multicolumn{3}{|l|}{ Viscosity in mpa.s } \\
\hline Initial & $245.00 \pm 1.73$ & $713.67 \pm 2.08$ \\
\hline After addition of $0.5 \% \mathrm{AlCl}_{3}$ & $213.33 \pm 2.08$ & $640.67 \pm 2.08$ \\
\hline$\%$ change & $\downarrow 12.93$ & $\downarrow 10.23$ \\
\hline
\end{tabular}

However, these findings contradict the findings by ${ }^{6}$ which reported that addition of electrolytes such as sodium chloride increased viscosity of guar gum. This could probably mean that electrolytes have different effects on natural gums and it would not be possible to generalize on the effect of electrolytes on natural gums, therefore, treatment of the gums should be on individual basis. Viscosity decreased in the order $\mathrm{AlCl}_{3}>\mathrm{CaCl}_{2}>$ $\mathrm{NaCl}$. This observation agreed with the Schulze-Hardy rule which states that the valence of electrolytes having a charge opposite to that of the hydrophobic particle determines the effectiveness of the electrolyte in aggregating the particles.

Pharm \& Biosci J. 2020: 8(2); 4 
Type and concentration of electrolytes present naturally in tap water used for reconstitution of dry suspensions may affect the viscosity the suspension. Decrease in viscosity could possibly be due to slowing down the hydration of gums used as suspending agents causing inadequate viscosity. Therefore, where possible, it is appropriate to use deionized water during reconstitution of dry suspensions to avoid alteration of viscosity.

\subsection{Effect of aging on viscosity of paracetamol suspension}

Table 5 shows the effect of aging on viscosity of paracetamol suspensions formulated using okra gum and tragacanth gum particles of undersize $180 \mu \mathrm{m}$. At day one, the viscosity of the suspensions containing okra gum and tragacanth gum was 244.0 \pm 6.25 and $745.65 \pm 6.43$ respectively. At five (5) weeks, the viscosity had dropped to $210.33 \pm 1.15$ and $707.33 \pm 3.51$ respectively indicating a decrease of $13.80 \%$ and $5.14 \%$ respectively. The viscosity of the suspension formulation containing okra gum dropped more than that of the suspension formulation containing tragacanth gum.

Table 5: Effect of Aging on Viscosity of Paracetamol Suspension

\begin{tabular}{lccc}
\hline \multirow{2}{*}{$\begin{array}{l}\text { Formula } \\
\text {-tion }\end{array}$} & \multicolumn{3}{c}{ Viscosity in mpa.s ( at RTP) } \\
\cline { 2 - 4 } & Day $\mathbf{1}$ & 5 weeks & \% change \\
\hline S1 & $244.00 \pm 6.25$ & $210.33 \pm 1.15$ & $\downarrow 13.80$ \\
S2 & $745.67 \pm 6.43$ & 707.33 & $\downarrow 5.14$ \\
\hline Mean \pm SD ( $=$ = 3 determinations $)$ & &
\end{tabular}

\section{Conclusion}

The viscosity property of okra gum in paracetamol suspension was susceptible to type of electrolyte, $\mathrm{pH}$, temperature and aging and thus formulators and caregivers should consider these factors when designing or using pharmaceutical suspensions containing natural gums such as okra and tragacanth as viscosity enhancing agents. The study implies that when reconstituting dry suspensions that have been formulated using natural gums such as okra gum, where possible, it is appropriate to use deionized water, reconstitute and store within the recommended temperature to avoid alteration of viscosity which may affect the performance and bioavailability.

\section{Acknowledgment}

The author would like to acknowledge the African Centre of Excellence on Phytomedicine Research and Development (ACEPRD), University of Jos for the financial support.

\section{Conflict of interest}

Nil

\section{Author's contributions}

VON came up with the idea, conducted literature review, designed and carried out data collection, and drafted the manuscript. JIO offered guidance during development of the idea and data collection, and reviewed the manuscript. All the authors read and approved the final copy of the manuscript.

\section{References}

1. Audu-Peter JD, Kadiri JO, Gyang JS. Effects of heating on the viscosity and rheology of Grewia mollis, Bombax ceiba and their binary mixture. Journal of Pharmaceutical and Allied Sciences. 2011; 8 (1): 12891297.

2. Gbenga LB, Oluwabunmi A. Suspending properties of natural gums extracted from Abelmoschus esculentus pod and Chrysophyllum albidium fruit. African Journal of Pharmacy and Pharmacology. 2015; 9 (10): 321 326.

3. Murthy NP, Devi VM, Sahoo KS, Mahapatra KA, Khandai M. Evaluation of sedimentation stability in paracetamol suspensions with Plantago ovata mucilage as suspending agent using near-infrared transmission measurements. Der Pharmacia Lettre. 2015; 7 (7): 85-96.

4. Venkateswarlu K, Chnadrasekhar KB, Ramachandra R. Development and in-vitro evaluation of reconstitutable suspension of flucloxacillin. Marmara Pharmaceutical Journal. 2016; 20 (3): 280-287.

5. Ogaji IJ, Hoag WS. Effect of grewia gum as a suspending agent on ibuprofen pediatric formulation. American Association of Pharmaceutical Scientists PharmSciTech. 2011; 12 (2): 507-513.

6. Mudgil D, Barak S, Khatkar SB. (2011). Guar gum: processing, properties and food applications-A Review. Journal of Food Science and Technology.2011; 51 (3): 409-418.

7. Ogaji I. Some physicochemical properties of acetaminophen pediatric suspensions formulated with okra gums obtained from different extraction processes as suspending agent. Asian Journal of Pharmaceutics. 2011; 5 (1): 15-20.

8. Aremu OI, Oduyela OO. Evaluation of metronidazole suspensions. African Journal of Pharmacy and Pharmacology. 2015; 9 (12): 439-450.

9. Farooq $U$, Malviya R, Sharma KP. Extraction and characterization of okra mucilage as pharmaceutical excipient. Academic Journal of Plant Sciences. 2013; 6 (4): 168-172.

10. Emeje M, Isimi C, Byrn S, Fortunak J, Kunle O, Ofoefule S. Extraction and physicochemical characterization of a new polysaccharide obtained from the fresh fruits of Abelmoschus esculentus. Iranian Journal of Pharmaceutical Research. 2011; 10 (2): 237-246.

Pharm \& Biosci J. 2020: 8(2); 5 
Nyandoro et al., Influence of Electrolyte Type, pH, Temperature and Aging on the Viscosity of Okra

11. Kadiri JO, Okafor IS. Evaluation of the suspending property of grewia gum in metronidazole suspension. Nigerian Journal of Pharmaceutical Research. 2010; 8 (1): $247-2547$. 